\title{
La apropiación en la comunicación y en la educación. Construcción teórica de la apropiación desde las experiencias comunicativas de estudiantes universitarios*
}

\author{
Appropriation in the Communication and Education. Theoretical Construction of the Appropriation from the \\ Communicative Experiences of College Students \\ A apropriação na comunicação e ensino. Construção teórica da apropriação desde as experiências comunicativas de \\ alunos universitários
}

\author{
Andrés Camilo Agudelo Vergara ${ }^{\text {a }}$ \\ Universidad Tecnológica de Pereira, Colombia \\ camilo.agudelo@utp.edu.co \\ ORCID: http://orcid.org/0000-0001-8636-9543
}

DOI: https://doi.org/10.11144/Javeriana.syp38-75.acec

Fecha de recepción: 31 Agosto 2018

Fecha de aprobación: 30 Junio 2019

Fecha de publicación: 15 Noviembre 2019

\section{Resumen:}

Este artículo evidencia las primeras reflexiones teóricas de una investigación en curso acerca del concepto de apropiación en los estudiantes de la Licenciatura en Comunicación e Informática Educativa. Dadas las densidades comunicativas de las culturas mediáticas actuales, resulta pertinente buscar un concepto que no solo se relacione con la recepción, sino también con la interacción. Se emplea una metodología cualitativa e interpretativa, tomando elementos de la Teoría Fundamentada para construir dimensiones teóricas de la apropiación en el contexto observado. Estas dimensiones son: problemáticas de sentido, interpelación, interpretación e interacción. Se encontró, en esas dimensiones, que los lineamientos teórico-curriculares de la Comunicación en la Licenciatura han generado incertidumbres en los estudiantes relacionadas con sus perfiles profesionales. Esta contingencia, a la vez apertura, ha posibilitado que produzcan apropiaciones significativas con medios y tecnologías. Estas primeras reflexiones ofrecen un marco de análisis interpretativo para repensar las culturas mediáticas y la educación en tecnologías.

Palabras clave: apropiación, problemáticas de sentido, interpelación, interpretación, interacción.

\section{Abstract:}

This article provides the first theoretical reflections of an ongoing research on the concept of appropriation in the students in the Communication and Educative Informatics undergraduate program. Due to the communicative densities in nowadays media cultures, it is pertinent to find a concept not only related to the reception, but also to the interaction. A qualitative and interpretative methodology is used but taking elements from the Grounded Theory in order to build theoretical dimensions of the appropriation in the observed context. These dimensions are: sense problems, interpretation and interaction. It was found that for these dimensions the theoretical-curricular guidelines of the said undergraduate program have brought about some uncertainties in the students related to the professional profile. This contingency -which is also an opening- has enabled the production of significant appropriations with the media and technologies. These first reflections provide an interpretative analysis framework to rethink the media culture and the education in technology.

Keywords: appropriation, sense problems, interpellation, interpretation, interaction.

\section{Resumo:}

Este artigo demonstra as primeiras reflexões teóricas de uma pesquisa em andamento acerca do conceito de apropriação nos alunos da Licenciatura em Comunicação e Informática Educacional. Dadas as densidades comunicativas das culturas midiáticas atuais, resulta pertinente procurar um conceito que não apenas está relacionado com a recepção, mas também com a interação. Usou-se uma metodologia qualitativa e interpretativa, tomando elementos da Teoria Fundamentada para construir dimensões teóricas da apropriação no contexto observado. Essas dimensões são: problemáticas de sentido, interpelação, interpretação e interação. Encontrou-se, nessas dimensões, que as diretrizes teórico-curriculares da Comunicação na Licenciatura geraram nos alunos incertezas relacionadas com seus perfis profissionais. Esta contingência, ao mesmo tempo apertura, possibilitou a produção

Notas de autor

a Autor de correspondencia. Correo electrónico: camilo.agudelo@utp.edu.co 
de apropriações significativas com meios e tecnologias. Estas primeiras reflexões oferecem um quadro de análise interpretativa para repensar as culturas mediáticas e o ensino em tecnologias.

Palavras-chave: apropriação, problemáticas de sentido, interpelação, interpretação, interação.

\section{Introducción}

El 30 de octubre de 1938, la radio estadounidense emitió la noticia de una invasión extraterrestre a nuestro planeta. Se decía que provenían de Marte, que sus intenciones no parecían ser muy amigables y que, inclusive, ya habían provocado algunas explosiones en diferentes partes de Nueva Jersey. Durante la transmisión, un número inexacto de ciudadanos americanos salieron de sus casas a buscar un refugio seguro, mientras otros corrían por las calles buscando a la policía (Cantril, 1979). Esta noticia había causado pánico social y también controversia sobre su falsedad o veracidad. El autor de este caos masivo fue Orson Welles, quien adaptó la novela de "La guerra de los mundos", escrita por Herbert George Wells, en formato radial noticioso. Su intención era recrear la experiencia de una invasión extraterrestre en los oyentes.

Los ciudadanos recibieron esta historia como si fuera una noticia y por eso vivirían un conjunto de emociones cruzadas como el miedo, la indignación y, quizás, el encanto. Lo cierto es que para cada emoción habría un tipo evidente de acción social. Para el miedo habría pánico y desorden público, y para la indignación y el encanto habría una controversia mediática post-narración (Cantril, 1979). Los estudios de comunicación en esa época resaltaban, con este eventual acontecimiento, que entre los medios y la acción social había una relación determinista de tipo causa-efecto. El concepto de recepción adquirió esa lógica lineal y, al verla en la práctica, se distanció fenomenológicamente de los medios.

Más de setenta años después, las condiciones tecnológicas y mediáticas hacen que ese asunto de las recepciones sea mucho más complejo. Hoy en día, las tecnologías de comunicación son heterogéneas, así como sus participantes, sus contenidos y sus acciones. Los medios y tecnologías de la información y la comunicación (TIC) se han fundido en las experiencias cotidianas. Ahora es muy difícil saber el modo en que un medio de comunicación incide en la acción social cuando esta última de por sí ya está mediatizada. Se asiste, en este sentido, a unas densidades comunicativas que demandan urgentemente replantear las perspectivas deterministas de la recepción.

Para lo anterior, se busca un concepto más adecuado que pueda incorporar no solo la recepción, sino también la interacción, es decir, la densificación comunicacional de las culturas mediáticas actuales. Tal vez, si se comienzan a observar las prácticas sociales con medios de comunicación, como si fueran formas de apropiación más que de recepción, sería posible tener un diagnóstico más contextualizado de los fenómenos mediático-culturales.

Por lo tanto, en este artículo se plantea cómo la apropiación, entendida como marco conceptual en la relación entre medios y acción social, puede posibilitar una observación holística continente de audiencias, usuarios, lenguajes, tecnologías y medios de comunicación. Ahora bien, si las prácticas mediáticas ya no son definidas estrictamente como recepciones, sino como apropiaciones, entonces estas pueden también ser entendidas como prácticas creativas, constructivas e interpretativas por parte de la audiencia. En síntesis, el enfoque de la apropiación toma en cuenta las formas simbólico-culturales construidas en el contexto popularmediático (Martín-Barbero, 1987), que son tenidas en cuenta en las perspectivas interpretativas. De esta manera, lo que se quiere analizar aquí es cómo cobra sentido teórico el concepto de apropiación cuando la mirada se desplaza de una línea determinista a un círculo interpretativo, en el que algunos investigadores hispanohablantes de la comunicación y la educación han venido apostando y teorizando las últimas tres décadas (Aparici, 2010).

Para hacer la reflexión de la apropiación desde una perspectiva interpretativa, se decide observar las experiencias mediáticas de los estudiantes de la Licenciatura en Comunicación de Informática Educativa 
(LCIE) en la Universidad Tecnológica de Pereira (UTP). Estas experiencias de formación no solo se localizan en aulas de clase e informáticas, sino también en entornos sociales mediatizados tipo online, en espacios públicos y en sectores privados donde realizan prácticas pedagógicas, periodísticas, investigativas y analíticas de lenguajes mediáticos; lugares en los cuales experimentan, piensan y analizan sus horizontes como futuros LCIE. Por lo anterior, este contexto se convierte en un referente clave para construir conceptualmente la apropiación y, en el orden inverso, esta construcción va arrojando pistas para comprender en ellos sus prácticas comunicativas y mediáticas en la vida académica que desbordan los procesos de recepción.

\section{Referente teórico}

El referente teórico se enmarca en dos aspectos. El primero es el contexto cultural de la apropiación, un contexto que inevitablemente pasa por estéticas de entretenimiento, de gustos populares, de nuevas subjetividades y de prácticas políticas, todas ellas mediadas por tecnologías digitales de comunicación. Estos elementos constituyen el contexto denominado por los autores más contemporáneos de la comunicación como Culturas Mediáticas (Martín, 2003; Rincón, 2006). Estas culturas se caracterizan por producir rituales que convierten los medios en experiencias cotidianas, prometer expresión social a través de los diversos formatos mediáticos y reivindicar las nuevas sensibilidades que posibilitan la construcción de otras realidades políticas y sociales (Rincón, 2006).

Ese contexto no es ajeno a los estudiantes en mención. En medio de sus deberes académicos, ellos desarrollan habilidades expresivas mediadas por tecnologías con el ánimo de reivindicar y reconstruir constantemente sus subjetividades. Por eso, se entiende que sus prácticas comunicativas las hacen, a pesar de ser universitarias y académicas, en el marco de las culturas mediáticas. En efecto, las fronteras entre lo académico y lo no-académico en la LCIE se diluyen constantemente en sus acciones, así como también lo hacen las culturas mediáticas en general con relación a las fronteras entre la política y el entretenimiento.

El segundo aspecto hace referencia al enfoque interpretativo de la comunicación mediática, tomado de autores como Jhon Thompson y Clifford Geertz. La recepción es vista aquí como una actividad y no como algo pasivo (Thompson, 1998), puesto que el campo de análisis de la recepción no recae en el contenido simbólico en sí, sino en el contexto cotidiano en el que ocurre dicha actividad. A partir de este cambio en la mirada, generalmente la apropiación se entiende como una característica cultural generadora de símbolos significativos y sentidos de mundo desde las expresiones que relucen en lo cotidiano (Geertz, 2003), basada en procesos activos de recepción e interacción social.

En efecto, la interpretación comienza por la acepción más formal del concepto de apropiación. Inicialmente, se relaciona con hacer algo propio de alguien y, al mismo tiempo, tomar algo para sí y adueñarse de ello (Real Academia Española [RAE], 2014). Pero la apropiación no termina en esta noción de propiedad. Al hacerlo suyo, lo combina con los otros elementos adquiridos en otras fuentes, transformando lo que inicialmente no era de sí. De este modo, la propiedad no es heredada, sino construida a partir del acto de coger algo y combinarlo con otros elementos. Es por eso, además, una propiedad creada con referentes propios y de otros que no eran suyos, pero que ahora lo son por medio de la unión de los mismos.

De esta manera, puede entenderse la apropiación como ese proceso de creación de algo con elementos de otros, y tomar el resultado como suyo. Inclusive, desde el arte se han hecho obras derivadas de otras, caracterizadas dentro del movimiento artístico del apropiacionismo (Santiago Martin de Madrid, 2011). En este sentido, es posible afirmar que lo que Kottack alguna vez llamó como "aprendizaje cultural", esté muy asociado a la apropiación en la medida en que se refiere a los sistemas simbólicos que son "absorbidos" y apropiados gradualmente por las personas para definir su mundo y expresar sus juicios (Kottak, 2011). Sin embargo, el aprendizaje cultural se sigue asociando con mayor fuerza a lo adquisitivo; en cambio, la apropiación, con lo anteriormente dicho, recoge un sentido más creativo-interpretativo. 
Esta perspectiva, con sus dos aspectos antes mencionados, resulta eminentemente antropológica, y funge para reconocer en la realidad social de los estudiantes las categorías propias, de tipo cultural, de sus modos de apropiación en las que articulan las TIC y los medios de comunicación.

\section{Horizonte metodológico}

Dado que el objetivo principal consiste en reflexionar y describir las características de la apropiación, se busca una metodología que pueda tratar interpretativamente los datos del contexto social y educativo de los estudiantes, de tal forma que con este tratamiento se logre llegar a comprensiones teóricas del tema. Por eso, se trabajó el análisis desde la metodología cualitativa de la Teoría Fundamentada, la cual se refiere generalmente a un conjunto de métodos basados en dejar aparecer la teoría desde la misma realidad: "comienza con un área de estudio y permite que la teoría emerja a partir de los datos” (Strauss y Corbin, 2002, p. 22).

Los datos para la construcción teórica no solo comprenden el conjunto de experiencias sociales de los estudiantes dentro de la Universidad, sino también el aspecto curricular que, de algún modo, ha sido un estimulante significativo en sus iniciativas de participación comunicativa. Participaciones que, a propósito, han densificado las articulaciones entre medios, tecnologías y acciones. Con relación a este currículo, se toman, particularmente, los contenidos de las asignaturas teóricas de comunicación de la LCIE, sus propuestas reflexivas y sus alcances educativos.

\section{Reflexión metodológica}

Durante los últimos dos años, las dinámicas académicas de los estudiantes han sido observadas con mayor detenimiento que los años anteriores. Empezando por el aula -en las clases teóricas de comunicación orientadas desde primero hasta séptimo semestre- lugar en el cual se han recogido experiencias de aprendizaje sobre la comunicación, la sociedad, la cultura, las tecnologías y los medios. Consecutivamente, se recogieron datos en pasillos, cafeterías y zonas de esparcimiento, relacionados igualmente con experiencias, pero no tanto de aprendizaje, sino más de diálogo y esparcimiento. Se acudió a métodos conversacionales de observación participante, tales como entrevistas y tertulias; y, finalmente, se tuvieron en cuenta las dinámicas expresivas y comunicativas que hacían en medios digitales.

Los criterios conversacionales, así como de selección de contenidos simbólicos a analizar en entornos digitales, eran abiertos. Por eso fueron observaciones y conversaciones ubicadas en la dinámica de la espontaneidad. En efecto, desde la teoría fundamentada se piensa en construir el concepto-teoría desde en una actitud abierta de observación de la realidad social, con la cual se inicia un proceso de codificación que, así mismo, es denominado codificación abierta, que consiste en el primer nivel de selección y comprensión de la realidad. Esta permite seleccionar, descartar, limitar y dar coherencia a los datos, y deja como resultante un conjunto de conclusiones teóricas preliminares de lo observado (Strauss y Corbin, 2002).

\section{Generalidades de la apropiación}

Primero, se identificó que los estudiantes acceden fácilmente al uso de estas tecnologías, no precisamente porque el manejo de estos dispositivos haga parte de su competencia formal en la carrera, sino porque, en medio de estos medios, tanto en las narrativas y formatos, como en los demás productos simbólicos mediatizados creados por las ciberculturas y las industrias culturales ${ }^{1}$, ellos encuentran respuestas significativas, así sean temporalmente cortas, a un conjunto de incertidumbres referentes a sus vidas, a sus proyectos personales y a sus futuros profesionales. Estas incertidumbres se han evidenciado recurrentemente 
en los estudiantes, y se han definido aquí como problemáticas de sentido, que serán comentadas con más detalle en la siguiente sección.

Así mismo, casi todas sus opiniones y cuestiones sobre sus vidas, la ciudad, la política, la educación y el arte, están atravesadas de algún modo por los medios y las TIC. Estas mediatizaciones cotidianas se desarrollan a partir de la producción de contenidos simbólicos digitales en formato de imagen, sonido, video y texto, replicados con alta densidad en las diferentes plataformas sociales digitales. La articulación es tan densa, y la actividad comunicativa tan álgida, que resulta inadecuado hablar de cotidianidad y de TIC como si esta última no se hubiera fundido, sin desaparecer, en la primera.

A pesar de esto, se encontró que en esas densidades hay tres dimensiones claves, entendidas aquí como habilidades de las culturas mediáticas: la primera es la habilidad de sentir; la segunda, la de leer; y, por último, la de actuar. Ahora, el desarrollo de estas habilidades depende necesariamente del grado de profundidad de las problemáticas de sentido. Entonces, ¿cómo entender estas incertidumbres en relación con dichas habilidades en las culturas mediáticas?

\section{Incertidumbres: las problemáticas de sentido}

El caso de la emisión de la invasión de Marte, por ejemplo, persuadió a muchas personas no solo por lo novedoso del formato narrativo y a la incapacidad crítica conducente al miedo (Cantril, 1979, sino también por la incertidumbre y mística histórica que siempre se ha tenido acerca de las posibles vidas en otros planetas. Incertidumbre que sirvió como nicho para interpelar en modo de pánico social a miles de personas que escuchaban la emisión.

Esa incertidumbre es, precisamente, un ejemplo de una problemática, y de manera contundente se define acá como una problemática de sentido, puesto que manifiesta una duda o carencia histórica significativa que se cristaliza de varios modos y, en este caso, fue por medio del pánico social. Pudo haber sido otro tipo de interpelación diferente al pánico, pero se dio de tal manera debido a las características y propósitos del formato radial emitido. Lo que se rescata aquí es la capacidad de tocar las emociones de la audiencia gracias a ciertos aspectos arquetípicos y problemáticos de la cultura: la mística creada alrededor de los extraterrestres.

De este modo, el requisito en los jóvenes para sus prácticas mediáticas consiste en tener una o unas problemáticas de sentido, una crisis de representación que puedan resolver temporalmente en recepciones, producciones y prácticas digitales. Un ejemplo de una problemática general de sentido en las culturas mediáticas, y al mismo tiempo proyectada en los estudiantes de la LCIE, es el siguiente: los jóvenes de ahora están llamados a enfrentar y a asumir la incertidumbre de sus propias vidas, incertidumbres traducidas en crisis acerca de lo verdadero, lo válido y lo correcto. Paradójicamente, mientras más se asiste a una densificación de la información y el conocimiento, más se evidencia una ausencia de relatos que sienten certezas sobre aquellos viejos significantes como el amor, el trabajo, la familia, la identidad, la vida, el futuro, entre otros. Ante esta ausencia de relatos universales que puedan dar respuesta a estos interrogantes, la juventud ha empezado a encontrar historias, textos y pre-textos que puedan al menos ayudar a otorgar un sentido temporal a estos significantes vacios (Rincón, 2006), sin importar que, posiblemente mañana, estos significantes vuelvan a vaciarse y a producir nuevas búsquedas y problemáticas de sentido. Así, los jóvenes o usuarios de las culturas mediáticas no solo han aprendido a vivir en medio de la crisis y la contingencia, sino que también han sabido sobrellevar esta época gracias al uso y desuso constante de los diversos símbolos y relatos mediatizados en la comunicación mediática. Resuelven su presente en la inmersión de universos de sentido mediatizados, dotados de historias y narrativas cada vez más novedosas, de conocimientos, de actividades, de movimientos y de comunidades.

Este no es un concepto nuevo. Marc Augé llamó a estas problemáticas sobredimensionamiento de sentidos: "Lo que es nuevo no es que el mundo tenga, o tenga poco, o menos sentido, sino que experimentemos explícita 
e intensamente la necesidad cotidiana de darle alguno: de dar sentido al mundo, no a tal pueblo o a tal raza" (Auge, 1992, p. 35). Igualmente, Omar Rincón describe esta cultura como un escenario de significantes vacíos. Como se puede leer, esto no es contrario a los sobredimensionamientos de Augé: es tanta la densidad informacional, comunicativa y mediática, que los símbolos tradicionales se vacían de sentido, pierden el original y se mutan con otros sentidos recreados en relatos contemporáneos menos rígidos, menos genuinos y menos sublimes (Rincón, 2006).

A partir de esta reflexión, se irán resaltando algunas experiencias de los estudiantes que proyectarán de algún modo las problemáticas de sentido en las cuales van ejerciendo sus modos de sentir, leer y actuar.

\section{Modos de sentir: la interpelación}

Primero, los modos de sentir son de cierta forma interpelaciones que producen las TIC, es decir, llamados de atención. En las teorías clásicas de la comunicación de masas se hablaba de persuasión como forma de vínculo sensible entre los medios con la audiencia. Lo hacían desde una perspectiva notoriamente conductista y nuevamente determinista, pues se entendía que los medios y sus contenidos, especialmente la propaganda política, eran una especie de estímulos que producían ciertas respuestas/conductas sobre sus espectadores (Statera, 1979).

En el presente, cuando se incluyen los medios digitales en el panorama de la comunicación mediática, es necesario observar la interpelación, menos desde una perspectiva conductista y más desde una perspectiva estética que pueda detallar los tipos de sensibilidad que viven los espectadores y participantes de las culturas mediáticas. En los estudiantes, estas sensibilidades se desarrollan por medio de tres sensaciones: sensación de inmersión narrativa, sensación de identidad y sensación de socialidad.

\section{Sensación de inmersión narrativa}

"Tenemos que comprender que los medios de comunicación de masas, para ganar comunicabilidad e interacción social, deben contar de nuevo la vida desde lo emocional; fabular desde el entretenimiento" (Rincón, 2006, p. 13). Esta sensación de inmersión comienza en el amplio consumo de contenidos mediáticos de los estudiantes, como series, vídeos y noticias. La inmersión se da en el vínculo emocional con el contenido simbólico y su formato. Se vinculan significativamente a esas historias presentadas en las series, por ejemplo. Comentan sobre ellas, las ponen en discusión en clases, las convierten en chistes de pasillo; en fin, expanden el relato de diversas maneras, lenguajes y medios. Para poderlo expandir, se requiere insertarse en él, hacerlo parte de sus tramas cotidianas de vida. Ese es el proceso de inmersión narrativa. En efecto, también lo viven en las narrativas noticiosas de la política colombiana, especialmente lo que tiene que ver con el uribismo y la oposición ${ }^{2}$. Estas inmersiones han sido tan profundas que han expandido dichos relatos políticos en la centralidad de los contenidos del aula y de los temas diarios en sus redes sociales. Construyen expresiones políticas de alto nivel emocional, expanden estas noticias por medio de ellas y sus discursos se asocian al concepto de país. Todo esto ocurre especialmente en los medios digitales y lugares de esparcimiento en los que pueden interactuar con sus compañeros, docentes y con otros amigos de diferentes grupos sociales.

Gracias a estos procesos de inmersión narrativa en el escenario político, los estudiantes aprovechan sus vínculos emocionales con estas narrativas para recrear y resignificar críticamente el país en las clases de las asignaturas teóricas de la comunicación, especialmente en las que se tratan contenidos relacionados con política, tecnología y cultura. Las problemáticas de sentido que posibilitan estas inmersiones están vinculadas con las problemáticas de país en el orden de la votación por el plebiscito sobre los diálogos de Paz y, posteriormente, sobre las elecciones presidenciales del 2018. 
Es por esto que la inmersión narrativa no solo se produce con las historias estrictamente de ficción, sino también con los sucesos políticos y deportivos que, casi siempre, pasan por el sensacionalismo y el melodrama en algunos noticieros y diarios informativos, invitando así al espectador a insertarse políticamente en el asunto. De este modo, la inmersión narrativa obedece al acto de contar de nuevo, de narrar y representar la vida de tal manera que se impida al espectador ser neutral ante la historia.

Como se puede observar, la inmersión narrativa es la adhesión semántica a una realidad fabulada en y por los medios, y está fundamentada en la re-presentación sintetizada de la vida cotidiana. De esta manera, extrae lo más emocional de ella para renovarla en un formato diferente y novedoso, lo que produce un paisaje simbólico de acontecimientos, mundos y personajes que invitan constantemente al espectador a tomar partido, vivir y emocionarse respecto a la trama vista, oída y leída.

\section{Sensación de identidad}

Se ha visto cómo los estudiantes se identifican con ciertos políticos de oposición en el país. Estos personajes, desde los medios, expresan ciertos anhelos históricos que recaen como anhelos particulares en los estudiantes. Es decir que de la única manera que se puede lograr identificación con cierto contenido simbólico, en este caso un personaje político, es que haya previamente una ausencia de sentido particular de quien vive dicha sensación de identidad; problemática que se resuelve transitoriamente en un discurso político mediatizado y difundido viralmente en redes sociales digitales, en el que se incluyen opiniones personales de estudiantes que cuentan de otro modo, no sólo el contenido, sino también sus posiciones políticas al respecto.

Con lo anterior, esta resolución es un acto de complicidad, por un lado; y de producción narrativa, por otro. Se puede ser cómplice de la idiosincrasia de un personaje, de sus éxitos o fracasos, de sus debilidades o fortalezas, de su falta de autoestima, de su nivel de ego, de su estatus social, de su posición económica, entre otros. Se hace cómplice en la medida en que el espectador posee profundamente esas cualidades o defectos, o esas problemáticas. Esta complicidad satisface parcialmente al espectador, puesto que lo respalda, lo acompaña y hasta lo orienta a cómo hacer más llevaderas sus problemáticas cotidianas. De ahí, expande el relato en otros medios, sigue narrando y reconociéndose en la vida fabulada y contada (Montoya Londoño, 2010). Es esta la identidad establecida entre el espectador y el personaje u otros aspectos de la historia en general, es la manera en que los problemas personales y colectivos adquieren sentido, respuestas y significados a partir de la narración.

Se encontró que los modos de identificación de los estudiantes con los medios y sus contenidos parten casi siempre de sus problemáticas sobre el horizonte de sus perfiles profesionales. A partir de sus conversaciones cotidianas, tanto dentro del aula como fuera de ella, es evidente que los estudiantes manifiestan una cierta insatisfacción e incertidumbre sobre el quehacer de un LCIE. A partir de estas inquietudes, elaboran su perfil en la exploración de diversos medios y tecnologías, y logran sentir identificación en algunas derivas mediáticas, no sólo asociadas a ciertos personajes y tramas de series y películas, sino también, y sobre todo, a roles de producción tales como los de fotógrafos, diseñadores, productores audiovisuales, directores de cine y asesores educativos con tecnología. Las dudas se convierten en una constante búsqueda de identidad y, al mismo tiempo, en una construcción autónoma de su quehacer como futuro LCIE. Se han sentido interpelados por los medios y los contenidos curriculares debido, precisamente, a sus problemas significativos de identidad con el serprofesional.

Adicionalmente, se evidenció cómo las problemáticas de sentido se cruzan entre ellas y producen emergencias comunicativas diferentes. Por ejemplo, sus problemáticas en el orden de lo político se relacionan constantemente con sus problemáticas sobre el quehacer profesional del LCIE. De ese modo, elaboran escenarios de comunicación política que se entretejen con la exploración de medios, lenguajes y plataformas para el ejercicio educativo del LCIE. 


\section{Sensación de comunidad y socialidad}

Tanto los temas políticos como los temas centrales de la carrera ponen a los estudiantes en una preocupación colectiva; es decir, los inserta en algo que no solo es individual sino de muchos, les otorga el escenario problemático adecuado para que se sientan vinculados en algunas comunidades temáticas en las que ejercen opinión. Sienten que sus expresiones son oportunas debido a la noción previa de que estos mensajes convocan a un público que también está generando opinión sobre lo mismo. Este sentir se ha denominado acá como sensación de socialidad.

Esta preocupación colectiva se tensiona entre ser una unidad superior hegemónica que imposibilita la diferencia, puesto que la polarización política en Colombia no ha permitido en muchos casos que los estudiantes tengan argumentos disímiles con respecto al antagonismo manifestado en sus opiniones, por un lado; y entre ser una problemática potencial de sentido que produce, en efecto, diversas derivas de opinión y proposición en los estudiantes, por el otro. Las dos problemáticas, a pesar de todo, producen contexto social participativo, en el cual se establece una socialidad alejada del individualismo y el extremo colectivismo alienado. Una socialidad cercana teóricamente a la noción de mundo intersubjetivo (Schutz y Luckmann, 2001).

En los medios, la expresión de uno posibilita al otro vivir una sensación de pertenecer a algo más grande que su familia y amigos, posiblemente a una comunidad nacional, pero también a comunidades específicas de gustos, hobbies y partidos políticos. Es quizás una sensación fundamental que promueve la participación social, la intersubjetividad y la vinculación del sujeto con un mundo en el cual es un contribuyente de la construcción de la cultura a la que pertenece. De ahí que los estudiantes participen activamente en diversos medios de comunicación, puesto que estas incertidumbres producen valores de cohesión, discusión y comunidad. Son problemáticas comunes que los convoca constantemente al debate, y, en ese sentido, manifiestan una sensación de comunidad.

A partir de estos tres tipos de sensaciones, las culturas mediáticas expanden historias, recrean relatos y noticias, experimentan con medios y tecnologías, se enriquecen de contenidos y de significados con los cuáles se identifican para comunicar y tejer comunidad. Desde una perspectiva educativa, no es posible plantear propuestas sostenibles de enseñanza y aprendizaje con medios de comunicación si no se comprenden y aprovechan las interpelaciones en las que viven constantemente los jóvenes contemporáneos.

\section{Modos de leer: la interpretación}

Los modos de leer no son precisamente los aprendizajes adquiridos de los contenidos curriculares, sino los signos construidos sobre estos contenidos en relación con sus experiencias fuera del aula; es decir, interpretaciones, creaciones sígnicas que se relacionan con otras y que les posibilita estar en el mundo. "El signo apela a un estar-en-el-mundo específicamente 'espacial', y no puede ser verdaderamente 'comprendido' como signos si nos limitamos a mirarlo y constatarlo como una cosa señaladora que estuviera allí" (Heidegger, 2003, p. 100). La interpretación es, entonces, la acción por la cual el ser le da sentido al mundo por medio de un proceso llamado significación, el cual, diría Heidegger, no puede desprenderse del contexto en el que emerge dicho proceso. Esto no es ajeno a los mensajes mediáticos. Thompson (1998) describe este proceso de la siguiente manera: "El individuo que recibe un producto mediático debe, de alguna manera, prestarle atención (leer, mirar, observar, escuchar, etc.); y, al hacer esto, el individuo comúnmente se ve ocupado en dar sentido al contenido simbólico transmitido por el producto" (p. 65).

Debido a que en los estudiantes viven generalmente problemáticas de sentido referidas a la indeterminación laboral y profesional del perfil del LCIE, por un lado; y a la situación política del país, así como su norte, por otro, se producen las condiciones necesarias para crear sentidos a estas incertidumbres a partir de prácticas 
mediáticas. Utilizan la ironía política, por ejemplo, desde lenguajes audiovisuales espontáneos y meméticos por redes sociales digitales que, además de hacer campaña y contracampaña política, funcionan como modo de significación de estas problemáticas. Así mismo, otorgan significados desde la parodia y la ironía a los significantes vacíos de país, y, en el mismo orden, construyen significativamente un perfil de LCIE enriquecido de prácticas de comunicación política y de movilización simbólica mediante el uso expresivo de la fotografía, la informática y la producción televisiva.

Además de lo anterior, algunos estudiantes responden a las cuestiones del perfil con prácticas de producción audiovisual de series web; otros, con producción de cine; y otros, con propuestas pedagógicas en el uso de TIC.

De ahí que se considere como un proceso activo de construcción más que de adherencia de una información. Por esa misma razón, la apropiación no se queda con el "tomar de", sino que continúa en el "crear a partir de". Es un proceso creativo de uso de símbolos, que Clifford Geertz (2003) denomina construcción:

Mientras vive los utiliza, o utiliza algunos de ellos, a veces deliberadamente o con cuidado, lo más frecuentemente de manera espontánea y con facilidad, pero siempre lo hace con las mismas miras: colocar una construcción sobre los sucesos entre lo que vive para orientarse dentro del 'curso en marcha de las cosas experimentadas', para decirlo con una vívida frase de John Dewey. (p. 52)

Estas construcciones, dado que están en función de orientar la experiencia, consisten en formas de sentido que auxilian, así sea temporalmente, las incertidumbres del hombre.

Lo que se puede observar generalmente en los estudiantes es que las mismas condiciones de apertura significativa y de incertidumbre, y de experimentación mediática y tecnológica que arroja la carrera de la Licenciatura en cuestión, les ha permitido elaborar de forma más autónoma sus criterios propios sobre el quehacer del LCIE. Y si esta afirmación se extrapola a las condiciones más generales de las culturas mediáticas, se puede evidenciar que allí también hay prácticas experimentales con medios y lenguajes en el orden de la variedad, la diferencia y la diversidad. Generalmente, obedecen a ciertas problemáticas de sentido que los interpela y los vincula a la interpretación constante de sus vidas.

En conclusión, el proceso creativo de significación-interpretación se fundamenta en la interpelación y, a su vez, en la problemática de sentido. Sin su vida cotidiana como problema, en los estudiantes no habría interpelación y tampoco una interpretación densa y significativa alrededor de los medios de comunicación. Este es un proceso creativo en el cual es difícil saber dónde, cómo y cuándo va a terminar. Es por eso que la interpretación es contingente y emergente. Solo se puede saber el antes más que el después, por lo cual se puede decir que está condicionado por los rasgos históricos, sociales, políticos y económicos de la problemática de sentido general de las culturas mediáticas, basada, como se había dicho antes, en la incertidumbre, en la crisis y en la búsqueda constante de respuestas ante el vaciamiento de sentido de los significantes universales del siglo pasado.

\section{Modos de actuar: la interacción}

Finalmente, los modos de actuar de los estudiantes no están determinados únicamente por el rol de aprendices. En efecto, su situación es la de ser agentes comunicativos, participantes de las interacciones dentro del aula, fuera de ella y en los contextos online. Estas comunicaciones se cruzan y se desajustan en cada lugar desde el punto de vista semántico, puesto que se habla de temas académicos en las redes, de temas cotidianos en el aula y en el campus, así como de política en los tres. De ahí que sus modos de acción sean, concretamente, interacciones.

Tanto las interpelaciones como las interpretaciones tienen lugar en lo común, es decir, en la comunicación. Es en las prácticas de interacción que se puede evidenciar cómo resuelven sus problemáticas de sentido. Los estudiantes dan lugar a sus significados en los escenarios de comunicación pública, como el aula de clase, las 
redes sociales y los actos culturales. Todas sus prácticas mediáticas relacionadas con la producción fotográfica, audiovisual y de opinión política recaen en estos escenarios públicos. Allí finaliza y toma su total forma la acción interpretativa, es decir, la acción de dar sentido.

Por tal razón, en las asignaturas teóricas, el aula se convierte en un escenario para discernir y concertar caminos acerca del perfil del LCIE, no solo a partir de prácticas estrictamente de comunicación oral, sino a partir de otros lenguajes ahora mediatizados. El proceso de apropiación mediática culmina y germina en la interacción, y en este sentido la LCIE cobra gran valor para la apropiación en los estudiantes, dado que ha brindado diversos escenarios abiertos para las interacciones, las discursividades y las prácticas mediatizadas. Si no hay interacción, es muy difícil hablar de apropiación. De ahí que el movimiento artístico del apropiacionismo cobre sentido en la exposición, en la publicación, en la comunicación, así como en el resto de dimensiones, más allá del arte, en las que el ser humano necesita ejercer el lenguaje y la expresión.

Thompson plantea que en la comunicación mediática hay tres tipos de interacción: la interacción cara a cara, la interacción mediática y la casi-interacción mediática. En la primera, hay varios factores como la copresencia, el diálogo, el uso de deícticos y de multiplicidad de señales simbólicas. En la segunda también hay procesos dialógicos bi-direccionales, aunque a partir de contextos espacio-temporales diferentes por parte de cada participante, dados por el uso de medios técnicos como papel, cables eléctricos, ondas electromagnéticas, etc. En la tercera, en la llamada casi-interacción mediática, los mensajes no son compartidos de forma bidireccional, sino de uno a muchos, es decir que posee un carácter monológico más que dialógico. Hay un grado de interacción más bajo que en los dos tipos anteriores; sin embargo, el autor no descarta que haya interacción debido al carácter social de la comunicación de masas:

Se trata de una situación estructurada en la que algunos individuos están implicados en la producción de formas simbólicas para otros que no están físicamente presentes, mientras que otros están fundamentalmente implicados en recibir formas simbólicas producidas por otros a los cuales no pueden responder pero con quienes pueden establecer lazos de amistad, afecto o lealtad. (Thompson, 1998, p. 116)

No necesariamente en las culturas mediáticas contemporáneas se debe acudir únicamente al segundo tipo de interacción. Realmente, sobre todo en los estudiantes, las tres formas de interacción se dan de manera imbricada, y todas son interdependientes. Thompson hace una separación de las tres formas solo a nivel conceptual, pues en las prácticas comunicativas de hoy, esas formas se correlacionan constantemente, a tal punto de no saber muy bien si a la ciudadanía consumidora de medios y tecnologías se les debería denominar usuarios en vez de audiencias. Este es un contexto de consumidores y productores, de receptores activos, de creativos, de comunicadores, de actores sociales pertenecientes a diversas comunidades online y offline. En estas interacciones los estudiantes han podido construir lazos, comunidades, movimientos, contextos sociales en general. Allí confluyen los significados, se producen otras problemáticas y, así, otras interpelaciones, lo que va enriqueciendo poco a poco los procesos de apropiación mediática.

Ahora bien, esto significa que la compleja interacción en estas culturas mediáticas pone en desafío a la semiótica, a la antropología y, en general, a los estudios de la recepción de medios. Desafíos relacionados con las implicaciones de la interacción en las formas de interpretación de los contenidos mediáticos, pero también con la interacción en sí misma, como práctica fundamental para la construcción de sociedad, política y ciudadanía. Hay que revisar cómo las culturas mediáticas se apropian de lo político y de la ciudad a partir de sus dinámicas comunicativas; y, en el caso de la escuela, cómo estas interacciones son efímeras y casi escasas en el aula de clase, lo que des-cotidianiza y des-conecta al educando de su sociedad y el contexto político en el que vive.

Es por ello que, en aras de analizar la apropiación como práctica educativa de las culturas mediáticas, la interacción debe ser pensada, al igual que la interpelación, desde el escenario de la sensibilidad y de la comunicabilidad, puesto que las interacciones no solo están dotadas de razones y argumentos, sino de identidades, caras, alianzas, sentimientos, emociones y procesos analógicos regulados por el carácter. De hecho, las culturas mediáticas son menos culturas de razón e ilustración y más de emociones, de comunidades 
y de entretenimiento (Rincón, 2006). La interacción no solo es la acción comunicativa habermasiana, de tipo deliberativa y crítica en búsqueda de la razón pública (Cortina, 2004), es también un ritual regulado por ciertas reglas de alianza y comunicación (Watzlawick, Beavin Bavelas y Don, 2002), y de factores de tipo sagrado y simbólico (Goffman, 1970).

Esta reflexión visibiliza qué tanto depende la educación de la comunicación. Así, se insiste que la comunicación es ese tipo de práctica de interacción humana antes mencionada -es decir, compleja y borrosa -, mediada por tecnologías e interfaces, pero, sobre todo, por sensibilidades, sueños e historias.

\section{La emergencia y su papel en la comunicación y la educación}

La comunicación pensada desde la apropiación es otra diferente a una lineal-determinista, una que pueda versar sobre los problemas y que posibilite la emergencia del signo, de la significación y del sentido en general. Así, toda comunicación, en el marco de la apropiación, debe posibilitar lo inesperado, asumir en cierto grado lo incier to y comprender la emergencia como una característica cualitativa de las formas sociales de las culturas mediáticas. De ahí que se tome con gran valor aquellos contextos que permitan la expresión social, tal y como el contexto de la LCIE lo hace. Las condiciones de apertura en la comunicación se hacen elementales a la hora de llegar a procesos de apropiación.

En las culturas más tradicionales de tipo occidental, por ejemplo, las normas religiosas tenían más peso sobre el pensamiento, la conducta y la opinión, así como la capacidad de reducir la expresión y la acción comunicativa. Era una sociedad más cerrada, la cual reprimía en cierto sentido la emergencia de otros puntos de vista acerca de la vida y de la existencia de dios. Ahora, al inicio de un siglo que está acompañado de un proceso de densificación de las prácticas comunicativas, al mismo tiempo en que se cristaliza el desafío por la interculturalidad, la solidez de las normas tradicionales de tipo religioso-occidental se tensionan, lo que ocasiona la emergencia de formas híbridas de religión y, en general, de la construcción de dios y de dioses.

En una sociedad política y culturalmente más abierta, la emergencia de prácticas comunicativas propicia más rápidamente transformaciones culturales, haciendo cada vez más difícil determinar y proyectar el futuro de esta. Solo queda asumir la contingencia y la posibilidad como formas insoslayables de comprender lo humano en esta problemática del no saber. Este es un presente nutrido de interpelaciones, interpretaciones e interacciones tan complejas que potencian nuevos horizontes de sentido indeterminables, de los cuales solo se pueden tener ciertas sospechas.

En efecto, la educación que está determinada y excesivamente planificada en sus metodologías priva de algún modo la apropiación. Los ejercicios de control neutralizan la emergencia. Esto impide la creación de lenguajes, de prácticas horizontales de opinión y de reconocimiento de problemáticas de sentido. Un modelo de educación y comunicación adecuado es aquel que promueva la interpelación, la interpretación y la interacción entre los agentes implicados en el acto educativo.

Actualmente, Latinoamérica vive estas tensiones entre la apertura y la censura comunicativa, que repercute en una preocupación por fortalecer la comunicación en los contextos educativos. De ahí emerge la necesidad de cruzar de forma teórica y práctica la educación con la comunicación, trabajo que empezó en este continente desde hace más de tres décadas (Aparici, 2010). La comunicación es educación cuando, en el sentido humano antes aclarado, se hace la praxis de la apropiación.

\section{Las tecnologías y los medios en las culturas mediáticas}

Ahora, ¿desde qué perspectiva entender las tecnologías digitales y los medios de comunicación que se han venido articulando en las prácticas cotidianas gracias a los procesos de transformación sociocultural? Tanto las posturas deterministas, en las que se consideran los medios y las tecnologías como autónomas y 
con capacidades intrínsecas de acción (Woolgar, 2005), como también las posturas románticas y escépticas reticentes a estos dispositivos (Argüello, 2010), son inadecuadas para entender la relación entre el hombre con la tecnología. En las culturas mediáticas, la relación es compleja, no lo era tanto cuando los medios tenían ciertas incidencias masivas en las audiencias, pero ahora, más que ver cuál es el orden lineal de causa y consecuencia, la perspectiva recae sobre la apropiación, es decir, sobre las formas simbólicas, expresivas, políticas y culturales en las que los medios están implícitamente articulados.

En efecto, si se redirige la mirada hacia la tríada entre interpelación, interpretación e interacción, es posible dar cuenta de la interdependencia entre el hombre con la tecnología. El artefacto, sus interfaces y sus productos simbólicos interpelan al usuario, pero esto no sería posible sin las condiciones antropológicas e historiológicas del hombre en las que este ha generado sus problemáticas de sentido. Con esta base cultural, el hombre cualifica el artefacto, le otorga un sentido, le asigna un significado, que luego resultará en modo de acción social y comunicativa con la misma y para los demás.

En este orden de ideas, se hace inadecuado generar discusiones sobre las potencialidades o amenazas de las tecnologías y medios o, en su defecto, sobre las potencialidades o amenazas de la comunicación mediática. Se trata, a este respecto, de llevar las discusiones hacia las características socioculturales de los diferentes contextos mediáticos y, en este caso, hacia los modos políticos, éticos, ontológicos y educativos de apropiación emergentes en los diferentes grupos culturales en los cuales los medios están presentes.

\section{Cierre}

La reflexión realizada durante el artículo tomaba como eje la preocupación acerca del modo lineal en que se observan los medios de comunicación, las tecnologías y la acción social. Se propuso, en este orden de ideas, entender estas relaciones en el contexto de las culturas mediáticas y de los estudiantes de la Licenciatura en Comunicación e informática educativa. De ahí se propuso un marco observacional diferente que parte de la categoría de la apropiación desde un enfoque interpretativo, y que pudiera permitir la comprensión, en otra lógica, de la relación que tiene el hombre con la tecnología.

Se evidenció que la apropiación es un modo de educación. Sin embargo, es un modo que está paradójicamente alejado de los modos de aprendizaje de las escuelas tradicionales, puesto que estas últimas no valoran la emergencia comunicativa en sus educandos y se exceden en la planificación y la prescripción de las prácticas de enseñanza y aprendizaje. De ahí que la apropiación no solo sea un marco sugerido para los estudios culturales de medios y tecnologías, sino también un detonante para la reflexión y el diseño de estrategias y políticas educativas tan urgentes, sobre todo, para el contexto latinoamericano.

La apropiación pasa de ser un concepto general a ser un marco conceptual transversal a la comunicación y a la educación en el contexto de las culturas mediáticas. Este marco no impide tomar una postura crítica frente a estas culturas en las que muchas veces se exceden en el consumo masivo de medios y tecnologías, hasta al punto en que, en algunas ocasiones, se produzcan lenguajes y prácticas de alto entretenimiento pero que, desde el punto de vista ético y político, dejan mucho que decir frente a la formación de públicos, audiencias, usuarios y consumidores.

Sin embargo, las pistas para esta inquietud quedan esparcidas en el mismo desarrollo de este texto: las formas más adecuadas para pensar en un horizonte político y ético más justo, más crítico, más dialéctico y más propositivo en las juventudes actuales, radica en re-direccionar las problemáticas de sentido genéricas, asociadas a las carencias ofrecidas por la sociedad del consumo, hacia las problemáticas más localizadas y situadas en los contextos más cercanos y cotidianos de los educandos - modelo educacional y comunicacional horizontal y con asunción de la emergencia-. Esto les posibilitaría, de algún modo, generar procesos de apropiación mediática en virtud de la transformación autónoma y significativa de sus modos de vida. 


\section{Referencias}

Aparici, R. (2010). Introducción: la educomunicación más allá del 2.0. En R. Aparici (coord.), Educomunicación: Más allá del 2.0. (pp. 9-12). Barcelona, España: Editorial Gedisa, S.A.

Ardèvol, E., y San Cornelio, G. (2007). "Si quieres vernos en acción: YouTube.com" Prácticas mediáticas y autoproducción en Internet. Revista Chilena de Antropología Visual, (10), 1-29.

Argüello, R. (2010). Las proyecciones de prometeo. Tecnologías de la Información y las Comunicaciones y la (trans)formación del Sujeto (1 ${ }^{\mathrm{a}} \mathrm{ed}$.) (Vol. 1). Bogotá: Fractalia Ediciones.

Auge, M. (1992). Los no lugares. Espacios del anonimato. Una antropología de la sobremodernidad. (trad. M. Mizraji). Barcelona: Gedisa, S. A.

Cantril, H. (1979). La invasión desde Marte. En M. Moragas (ed.), Sociología de la Comunicación de Masas (pp. 190-203). Barcelona, España: Gustavo Gili, S. A.

Cortina, A. (2004). Ciudadanía activa en una sociedad mediática. En J. Conill y V. Gozálvez (coord.), Ética de los medios. Una apuesta por la ciudadania audiovisual (pp. 11-31). Barcelona, España: Gedisa, S. A

Geertz, C. (2003). La interpretación de las culturas. Barcelona: Gedisa, S. A.

Goffman, E. (1970). Ritual de la interacción. Ensayos sobre el comportamiento de la cara. Buenos Aires: Ed. Tiempo Contemporáneo.

Heidegger, M. (2003). Ser y tiempo (trad. J. E. Rivera). Madrid: Trotta.

Kottak, C. P. (2011). Antropología Cultural (14a ed.). México, D. F.: McGraw-Hill.

Martín, M. V. (2003). Diásporas de sentido en torno a la cultura mediática. En F. Saintout (ed.), Abrir la comunicación. Tradición y movimiento en el campo académico (pp. 157-176). La Plata, Argentina: Ediciones de periodismo y comunicación.

Martín-Barbero, J. (1987). De los medios a las mediaciones. Barcelona: Gustavo Gili, S. A.

Montoya Londoño, M. (2010). Narracio\#n, emociones e identidad. Una lectura epistemolo\#gica y hermene\#utica. Desafios, 22(2), 277-303.

Real Academia Española (RAE). (2014). Diccionario de la lengua española (23a ed.). Madrid: RAE.

Rincón, O. (2006). Narrativas mediáticas. O cómo se cuenta la sociedad del entretenimiento. Barcelona: Gedisa.

Santiago Martin de Madrid, P. (2011). Apropiacionismo. Medios de comunicación de masas en las artes visuales. Recuperado de http://hdl.handle.net/10251/9580.

Schutz, A., y Luckmann, T. (2001). Las estructuras del mundo de la vida. Buenos Aires: Amorrortu editores S. A.

Statera, G. (1979). Las investigaciones sobre los efectos de los <mass media>. En M. Moragas (ed.), Sociología de la Comunicación de Masas (pp. 49-65). Barcelona, España: Gustavo Gili, S. A.

Strauss, A., y Corbin, J. (2002). Bases de la investigación cualitativa. Técnicas y procedimientos para desarrollar la teoría fundamentada. Medellín: Universidad de Antioquia.

Thompson, J. (1998). Los media y la modernidad. Barcelona: Paidós Ibérica.

Uribismo. (s. f.). Wikipedia, La enciclopedia libre. Recuperado de https://es.wikipedia.org/wiki/Uribismo\#Oposici ón_y_controversia.

Watzlawick, P., Beavin Bavelas, J., y Don D. J. (2002). Teoria de la comunicación humana. Barcelona: Herder.

Woolgar, S. (2005). Cinco reglas de la virtualidad. En Autor (ed.), ¿Sociedad virtual? Tecnología, 'cibérbole', realidad (trad. G. Galdón) (pp. 19-39). Barcelona, España: UOC.

\section{Notas}

1 Elisenda Ardèvol y Gemma San Cornelio tienen un trabajo antropológico denso en el que dan cuenta, no sólo conceptualmente, sino desde las experiencias de los jóvenes de Madrid, acerca de las relaciones políticas y los conflictos 
emergentes entre la cultura popular y las industrias culturales a partir de prácticas mediáticas (Ardèvol y San Cornelio, 2007).

2 Algunos elementos generales del partido uribista en Colombia y la oposición, pueden revisarse en (Uribismo, s. f.).

* Artículo de investigación.

\section{Licencia Creative Commons CC BY 4.0}

Cómo citar este artículo: Agudelo Vergara, A. C. (2019). La apropiación en la comunicación y en la educación. Construcción teórica de la apropiación desde las experiencias comunicativas de estudiantes universitarios. Signo y Pensamiento, 38(75). https://doi.org/10.11144/Javeriana.syp38-75.acec 